\title{
Acesso vascular para hemodiálise com cateter temporário de duplo lúmen em cães com insuficiência renal aguda
}

\author{
Hemodialysis vascular access with temporary double-lumen catheter in dogs with acute renal failure
}

\author{
Alessandra Melchert ${ }^{\mathrm{I}}$ Andre Marcelo Conceição Meneses ${ }^{\mathrm{II}}$ João Roberto de Araújo Caldeira Brant ${ }^{\mathrm{II}}$ \\ André Luis Balbi ${ }^{\text {III }}$ Jacqueline Teixeira Caramori ${ }^{\mathrm{III}}$ Pasqual Barretti ${ }^{\mathrm{III}}$
}

\section{RESUMO}

A hemodiálise é uma modalidade terapêutica que pode sustentar a vida do paciente com insuficiência renal aguda (IRA), enquanto este recupera a função renal. Para sua realização, é necessário estabelecer circulação extracorpórea, para que seja realizada a filtração do sangue, impondo a necessidade de um acesso vascular viável e eficiente. O objetivo deste estudo foi avaliar a eficiência e as complicações do acesso vascular para hemodiálise (HD), com cateter temporário de duplo lúmen inserido na veia jugular externa. Foram estudados 10 cães com IRA induzida por gentamicina, submetidos a sessões diárias de $H D$, com duração de uma hora, até a recuperação da função renal ou óbito. Foram realizadas 104 sessões de HD nos animais estudados, observando-se necessidade de troca do cateter em sete sessões (6,7\%), devido à obstrução do lúmen do cateter em seis sessões (5,8\%) ou por saída acidental do mesmo em uma sessão (1,0\%). Não se observou migração do cateter, infecção, hemorragia ou hematoma no local de entrada do cateter na pele, obtendo-se fluxo sanguíneo patente em 90,4\% das sessões. Concluiu-se que o acesso vascular na veia jugular externa com cateter temporário de duplo-lúmen mostrou-se viável, com ocorrência de poucas complicações, sendo, portanto, indicado como forma de acesso para a circulação extracorpórea para $\mathrm{HD}$ em cães com IRA.

Palavras-chave: acesso vascular, azotemia, complicações, hemodiálise.

\section{ABSTRACT}

Hemodialysis is a therapeutic procedure that can sustain the patient's life in acute renal failure (ARF), during the renal function recover. To perform hemodialysis (HD), an extracorporeal circulation is established to blood filtration, imposing the need of a viable and efficient vascular access.

\begin{abstract}
The aim of this study was to evaluate the effectiveness and complications of the HD vascular access with temporary doublelumen catheter inserted into the external jugular vein. Ten mongrel dogs with ARF, induced by gentamicin administration, were submitted to daily hemodialysis for one hour, until renal function recover or death. A total of $104 \mathrm{HD}$ sessions were performed. Catheter replacement was accomplished in seven sessions (6.7\%), due to catheter lumen mechanical obstruction in six sessions (5.8\%) or accidental catheter output in one session (1.0\%). No catheter migration, infection, bleeding or haematoma around the catheter insertion site was found. Effective blood flow rates were observed in 90.4\% HD sessions. In conclusion, the vascular access in jugular external vein with temporary double-lumen catheter was valuable, with few complications, and should be indicated to vascular access in extracorporeal circulation to $H D$ in ARF dogs.
\end{abstract}

Key words: azotemia, complications, hemodialysis, vascular access.

\section{INTRODUÇÃO}

A hemodiálise (HD) é um procedimento terapêutico que utiliza circulação extracorpórea do sangue do paciente, para que seja realizada filtração do mesmo através de uma membrana artificial semipermeável. O objetivo da HD é transferir toxinas e líquido em excesso do sangue do paciente para uma solução específica (solução dialisante) (COWGILL \& LANGSTON, 1996), aliviando a azotemia e os distúrbios metabólicos graves como os eletrolíticos, ácidobásicos, e acúmulo de líquidos e solutos tóxicos (LANGSTON et al., 1997).

\footnotetext{
'Universidade do Oeste Paulista (UNOESTE), Curso de Medicina Veterinária. Rod. Raposo Tavares, Km 572, 19067-175, Presidente Prudente, SP, Brasil. E-mail: alessandravet@unoeste.br. Autor para correspondência.

IPrograma de Pós-graduação em Fisiopatologia em Clínica Médica, Faculdade de Medicina, Universidade Estadual Paulista Júlio de Mesquita Filho (UNESP), Botucatu, SP, Brasil.

IIIDepartamento de Clínica Médica, Nefrologia, Faculdade de Medicina, UNESP, Botucatu, SP, Brasil.
} 
No homem, a principal indicação para realização da HD é a insuficiência renal crônica (IRC). Entretanto, em cães, a insuficiência renal aguda (IRA) é a indicação mais comum para a realização de diálise, também usada em intoxicações e sobredosagens de fármacos (VEADO et al., 2000). Sem diálise, os animais com IRA grave morrem freqüentemente devido às complicações da doença, antes que ocorra a recuperação da função renal. A HD aumenta a expectativa de vida desses animais, permitindo recuperação potencial (COWGILL \& ELLIOT, 2004) e reduzindo as taxas de mortalidade (FISCHER et al., 2004; MELCHERT, 2005).

Para realização da HD, é necessário estabelecer um circuito extracorpóreo. Um acesso vascular é necessário para transportar o sangue para o capilar de diálise e devolvê-lo ao paciente (COWGILL \& LANGSTON, 1996). Dentre as opções, há o shunt e a fístula artério-venosa, usados extensamente no homem (WEIJMER et al., 2004), porém pouco utilizados em Medicina Veterinária, sendo que os cateteres de duplo lúmen implantados em veia central, são a alternativa preferida para animais (COWGILL \& LANGSTON, 1996).

Dois tipos de cateter são disponíveis para realização de HD. Os cateteres permanentes com cuff (cateter tunelizado) são usados em pacientes que não apresentam outra forma de acesso vascular viável, sendo utilizados por longos períodos. Entretanto, um significante número de pacientes requer acesso vascular apenas temporário, como na presença de IRA. Nestes pacientes, o cateter temporário de duplo lúmen, também denominado cateter venoso central nãotunelizado, é utilizado, permanecendo viável por cerca de 30 dias (WEIJMER et al., 2004).

Em cães, o local de escolha para a implantação do cateter de HD é a veia jugular externa, utilizando-se principalmente cateteres temporários nãotunelizados (BONNET et al., 1994; FISCHER et al., 2004). Dentre os leitos vasculares usados para a HD no homem, o acesso pela veia jugular interna parece ter menos efeitos adversos (TESIO et al., 1994).

Complicações decorrentes do acesso vascular elevam a morbidade e mortalidade em humanos em esquema de HD (AGRAHARKAR et al., 2003). Infecções e trombose são os maiores problemas no acesso venoso jugular (BLAKE et al., 1990), além de obstrução do lúmen do cateter, sangramentos e hematomas (WOJCIESZEK et al., 2002). Em cães, os principais problemas encontrados incluem dificuldades na implantação do cateter, impedimento no fluxo sanguíneo, infecções (ELLIOT, 2000) e hemorragias (FISCHER et al., 2004).

Apesar de realizada com sucesso em cães nos Estados Unidos, no Japão e na Europa, a HD ainda tem uso limitado no Brasil. Um dos pontos a adequar é o acesso vascular, que deve fornecer fluxo sangüíneo eficiente para a circulação extracorpórea, de fácil manipulação, com mínimas complicações ao paciente.

O objetivo deste estudo foi então avaliar a eficiência do acesso vascular para hemodiálise em cães com IRA, utilizando-se o cateter de duplo lúmen nãotunelizado implantado na veia jugular externa, e observar as complicações decorrentes do procedimento.

\section{MATERIAL E MÉTODOS}

Após aprovação pelo Comitê de Ética em Pesquisa da instituição de origem, foram utilizados 10 cães, sem raça definida, adultos, idade entre um e cinco anos, machos, peso corporal entre seis e $20 \mathrm{~kg}$. Nestes animais, foi induzida Insuficiência Renal Aguda (IRA)por gentamicina $\left(\right.$ Gentocin $\left.^{\circledR}\right)$, com base no modelo experimental proposto por DANTAS et al. (1997). A HD foi instituída quando os cães apresentaram creatinina sérica acima de 5,0 $\mathrm{mg} \mathrm{dL}^{-1}$.

Previamente à primeira sessão de HD, os animais foram submetidos à colocação de cateter de duplo lúmen não-tunelizado (Two-Lumen Central Venous Catheterization Set ${ }^{\circledR}$, Arrow) de 8 French X 16 " $(16 \mathrm{~cm})$, de poliuretano. Este tipo de cateter apresenta dois lúmens em seu interior, chamados de vias arterial e venosa, que correspondem, respectivamente, ao lúmen por onde o sangue é retirado do corpo para filtração na máquina de HD e ao lúmen por onde o sangue já filtrado é devolvido ao paciente. Para implantação do cateter, foi realizada anestesia geral com propofol $\left(6,6-8,8 \mathrm{mg} \mathrm{kg}^{-1}\right)$ intravenoso, visando o tempo de anestesia entre $10 \mathrm{a}$ 15 minutos.

O cateter foi inserido preferencialmente na veia jugular externa direita, por via percutânea, após adequada tricotomia e anti-sepsia do local, conforme técnica descrita por MENESES et al. (2002), com uso de guia metálico e dilatador de vaso para acomodação do cateter. A porção extravascular do cateter foi fixada na região cervical lateral com cola do tipo cianoacrilato em gel (Superbonder ${ }^{\circledR}$, Loctite) (Figura $1 \mathrm{~A}$ ). Para verificação do correto posicionamento do cateter na veia cava cranial ou átrio direito, foi realizada avaliação radiográfica (Figura 1 B).

Para evitar a formação de coágulos nos lumens do cateter, estes foram lavados com solução fisiológica e preenchidos com heparina sódica (Liquemine $^{\circledast}$ - 25000UI $5 \mathrm{~mL}^{-1}$ - Roche), em volume proporcional a cada lúmen, sendo $0,7 \mathrm{~mL}$ na via arterial e $0,8 \mathrm{~mL}$ na via venosa, ao final de cada sessão de HD. Antes da sessão seguinte de HD, o cateter foi aberto, aspirando-se pelo menos $3 \mathrm{~mL}$ de sangue de cada lúmen, 


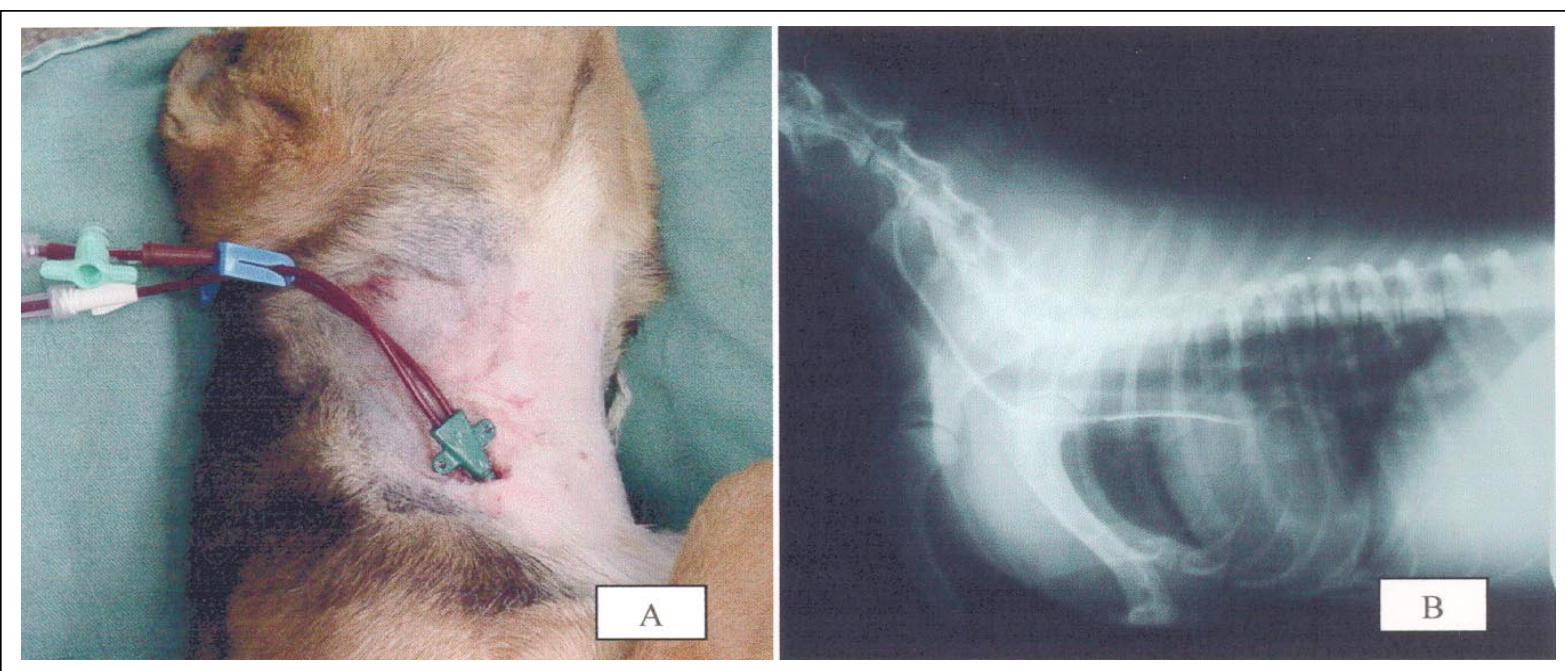

Figura 1- A) Cateter de duplo lúmen inserido na veia jugular externa direita de cão com insuficiência renal aguda nefrotóxica; B) Avaliação radiográfica do posicionamento cateter de duplo lúmen, localizado no átrio direito.

para remover a heparina. Foi realizada anti-sepsia do local de entrada do cateter e das vias arterial e venosa com iodo povidine e bandagem com pomada de Nitrofurazona $0,2 \%$ e atadura de gaze.

Realizou-se HD em aparelho de sistema proporcional com ultrafiltração controlada (System $1000^{\circledR}$ Series - Tina - Baxter Co.). Foram utilizados dialisadores capilares com membranas de polissulfona, área de superfície de $0,5 \mathrm{~m}^{2}$ (Hemoflow F3 - Fresenius polysulfone $^{\circledR}$ - Fresenius Medical Care). A solução dialisante foi composta por solução-tampão de bicarbonato de sódio a 8,4\% (F3 ${ }^{\circledR}$, Farmarin) e solução de eletrólitos (F3 ${ }^{\circledR}$, Farmarin) isenta de glicose, diluídas com água purificada, obtida por equipamento de osmose reversa portátil.

A anticoagulação durante a HD foi realizada com heparina sódica (Liquemine ${ }^{\circledR}$ - 25000UI $5 \mathrm{~mL}^{-1}-$ Roche), em bolus inicial de $100 \mathrm{U} \mathrm{kg}^{-1}$. O fluxo sanguíneo extracorpóreo foi restrito a 3 a $5 \mathrm{~mL} \mathrm{~kg}^{-1} \mathrm{~min}^{-1}$ nas duas sessões iniciais, para evitar excessivo clearance de uréia e desequilíbrio dialítico, e aumentado para 10 a $15 \mathrm{~mL}$ $\mathrm{kg}^{-1} \mathrm{~min}^{-1}$ a partir da terceira sessão. O fluxo da solução de diálise foi fixado em $500 \mathrm{~mL} \mathrm{~h}^{-1}$.

Foi determinado o tempo de coagulação ativado (TCA) de cada animal após o estabelecimento da IRA (TCA basal), bem como antes de cada sessão de HD, adicionando-se $2 \mathrm{~mL}$ de sangue a um tubo de ensaio contendo ativador (pó de vidro). A leitura foi realizada em monitor próprio (MCA $200^{\circledR}$ - Fundação Adib Jatene-SP), com resultados expressos em segundos (zero a 2000), que, em cães normais, varia de 41 a 80 segundos (BRANT, 2002).

Os animais foram dialisados diariamente, por uma hora, até o óbito ou recuperação da função renal, definida com a diminuição da creatinina sérica abaixo de $4,5 \mathrm{mg} \mathrm{dL}^{-1}$, por três dias consecutivos, melhora clínica e ausência de oligúria. As sessões de HD foram realizadas com os animais em vigília, não havendo necessidade de métodos de sedação para a contenção dos cães. Durante o procedimento, os animais foram mantidos em uma mesa, em decúbito esternal, lateral direito ou esquerdo, sem amarração e contidos manualmente por uma pessoa.

Os cateteres foram mantidos até o óbito ou durante 30 dias nos cães sobreviventes. Nos cães que não necessitavam mais de $\mathrm{HD}$, o cateter foi mantido realizando-se aspiração da heparina e substituição da mesma a cada três dias, seguida de curativo local com iodo povidine e bandagem com pomada de Nitrofurazona $0,2 \%$ e atadura de gaze. A retirada do cateter foi realizada pela tração do mesmo, seguida de compressão no local da punção durante 5 minutos.

Os cateteres danificados ou obstruídos durante o experimento foram substituídos e inseridos no mesmo local da punção inicial, com uso de guia metálico. Nos casos em que isso não foi possível, a inserção foi realizada na veia jugular externa contralateral.

Foram avaliados: dificuldades na implantação do cateter (venóclise, sangramentos e edemas); impedimento do fluxo sangüíneo durante a HD; obstrução mecânica ou dobradura do cateter; migração do cateter do sítio de instalação; exteriorização espontânea ou acidental do cateter; infecção no sítio de inserção do cateter na pele, classificada pela presença de edema, calor, dor, eritema e secreção purulenta; presença de febre ao início das 
sessões de HD; presença de hemorragia ou hematomas no local de inserção do cateter.

Avaliou-se também a contagem de leucócitos totais antes de cada sessão de HD e de plaquetas antes e ao final de cada sessão de HD. Estas variáveis paramétricas foram expressas como média e desvio-padrão e avaliadas pela análise de variância para medidas repetidas, seguida do teste de Newman Keulls para contraste entre médias. Foi considerado nível de significância de 5\% (MORRISON, 1990).

\section{RESULTADOS E DISCUSSÃO}

Realizou-se um total de 104 sessões de HD nos 10 cães. Cada animal recebeu entre 6 e 14 sessões de HD, até a recuperação da função renal ou óbito. Nos cinco cães sobreviventes (50\%), os cateteres foram removidos 30 dias após sua implantação na veia jugular externa, por tração seguida de compressão local, não se observando dor ou sangramento.

Dos 10 cães estudados, em nove foi possível a colocação do cateter em veia jugular externa direita, com punção da jugular externa esquerda em apenas um cão, no qual houve dificuldade na punção do lado direito. O tempo para instalação dos cateteres oscilou ente 9,4 e 15,3 minutos, não havendo necessidade de suplementar o propofol. Não foram observadas outras complicações decorrentes do implante do cateter, tais como sangramentos ou formação de edema no local da punção, enquanto observou-se dificuldade de punção em apenas uma implantação das 10 realizadas (10\%).

Foi observado um correto posicionamento do cateter na avaliação radiográfica de todos os animais estudados. Dos nove cateteres introduzidos pela jugular externa direita, oito apresentaram-se locados no átrio direito (Figura 1B), enquanto um deles e o único introduzido pela jugular externa esquerda estavam na veia cava.

Nas 104 sessões de HD realizadas, o fluxo sanguíneo foi adequado em 94 sessões $(90,4 \%)$, enquanto em 10 sessões $(9,6 \%)$ mostrou-se diminuído ou mesmo insuficiente para a realização de uma boa diálise. Em quatro destas sessões (3,9\%), o fluxo foi restabelecido após reposicionamento do cateter através de leve tração, aspiração dos seus lumens na tentativa de remover possíveis trombos ou coágulos e/ou inversão na conexão das linhas arterial e venosa de diálise, conforme descrito por ELLIOT (2000). Porém, houve necessidade de troca do cateter em seis sessões (5,8\%), devido à obstrução dos orifícios e/ou lúmen (cinco casos) ou dobradura (um caso). Não foi observada migração do sítio de instalação e, em uma sessão $(1,0 \%)$, ocorreu a saída acidental do cateter de duplo lúmen.
Houve, portanto, necessidade de troca do cateter em sete sessões de HD (6,7\%) nos 10 cães com IRA, sendo que em seis cães foi realizado um único implante (60\%), em dois cães (20\%) dois implantes e três implantes em dois cães (20\%). No cão com saída acidental, o novo cateter foi instalado na veia jugular contralateral esquerda, observando-se correto alojamento na veia cava. Nos casos de obstrução ou dano, a troca foi realizada pela introdução de guia metálico através do cateter danificado, para substituição do mesmo por um novo. Nestes cães, não houve necessidade de punção da jugular externa contralateral, conseguindo-se inserir o novo cateter no mesmo local da primeira punção; foi realizada anestesia com propofol em todas as substituições.

Não se observou infecção no local de entrada do cateter na pele. Porém, a avaliação baseouse apenas em dados clínicos e não incluiu cultura bacteriana. Do mesmo modo, não foram realizadas hemoculturas para comprovação de bacteremia ou sepse, avaliando-se apenas a temperatura corporal e a contagem total de leucócitos. Apenas um cão revelou aumento de temperatura corporal em uma sessão de $\mathrm{HD}$, sendo este animal um dos sobreviventes do estudo. As contagens de leucócitos não revelaram diferença estatística ao longo das sessões de HD e estiveram dentro dos valores de referência para cães (JAIN, 1993). Os valores médios ao início das sessões de HD oscilaram entre 7,9 $9 \pm 2,1$ (quarta sessão) e 14,4 $\pm 8,3$ (nona sessão).

Na avaliação do TCA, houve aumento significativo do mesmo ao final do procedimento em todas as sessões avaliadas, em relação aos valores basais individuais. Os valores médios do TCA foram de 93,9 $\pm 22,2$ seg para o TCA basal, enquanto que, ao final das sessões de HD, oscilaram entre 185,0 $\pm 30,8$

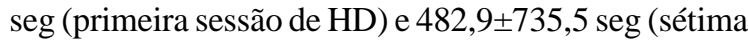
sessão) $(\mathrm{P}<0,05)$.

A contagem de plaquetas revelou diferença significante no período pós-dialítico (Pós-HD), em relação ao pré-dialítico (Pré-HD) em todos os momentos avaliados. Não foram observadas oscilações significantes na evolução diária, estando, entretanto, os valores médios, nos diferentes momentos, abaixo dos valores de referência para a espécie (JAIN, 1993). A contagem do número de plaquetas $\left(\times 10^{3} \mathrm{~mm}^{-3}\right)$ variou de 117,9 $\pm 46,0$ (quinta sessão) a 95,0 $\pm 64,2$ (nona sessão) (Pré-HD) e de 88,0 $\pm 46,0$ (sétima sessão) a 62,3 $\pm 25,1$ (segunda sessão) (Pós-HD).

Não foi observada a ocorrência de hemorragia ou hematomas logo após a implantação, durante o período de permanência dos cateteres ou durante as sessões de HD, período no qual era realizada anticoagulação do sangue com heparina. 
A implantação de cateter temporário de duplo lúmen em veia central é uma escolha favorável em cães, substituindo com sucesso a utilização de outros acessos vasculares (COWGILL \& LANGSTON, 1996). A utilização deste tipo de cateter apresenta benefícios diversos tais como praticidade e rapidez na implantação, possibilidade de uso imediato, baixa resistência venosa à circulação do sangue, apresentase indolor durante a sessão de HD e sua retirada é rápida e fácil (IKEDA \& CANZIANI, 2002), resultados corroborados por nosso estudo.

Atenção especial deve ser dispendida à implantação inicial e posicionamento correto do cateter de HD no átrio direito ou veia cava, localização preferida pelo maior fluxo sanguíneo disponível, o que permitirá adequado fluxo para a HD (ELLIOT, 2000), com menor incidência de colabamento do cateter com a parede da veia cava ou átrio, do que locado em vasos menores. $\mathrm{O}$ modo de implantação no presente estudo, conforme sugerido por MENESES et al. (2002), promoveu adequado posicionamento do cateter em todos os casos. A veia jugular se mostrou satisfatória para fornecer o fluxo de sangue para a HD, semelhantemente ao relatado por VEADO et al. (2000).

A inserção do cateter pela veia jugular direita é preferida pelo trajeto mais curto até a veia cava ou átrio direito, uma vez que a veia cava cranial se forma pela união da jugular externa à veia subclávia, e segue através do mediastino cranial à direita da traquéia, até chegar ao átrio direito (DICE et al., 1990).

O contato intermitente do cateter com a parede do átrio direito ou da veia cava pode impedir o fluxo sanguíneo. Das 10 sessões nas quais se observou problemas com fluxo, em quatro (40\%) este foi restabelecido após reposicionamento do cateter através de leve tração, aspiração dos lumens do cateter na tentativa de remover possíveis trombos ou coágulos ou inversão na conexão das linhas arterial e venosa de diálise, conforme descrito por ELLIOT (2000).

As principais complicações observadas em nosso estudo foram relativas à disfunção do cateter, por obstrução física por coágulos e aderência de fibrina $(4,81 \%)$ ou dobradura ( $0,96 \%)$. Segundo BESARAB \& RAJA (2003), quase todos os cateteres inseridos em veias centrais no homem desenvolvem coágulos de fibrina, entre uma a várias semanas após a implantação, até que ocorra obstrução. A incidência de trombose em cateteres percutâneos parece ser maior em cães e gatos que no homem, sugerindo diferenças nos mecanismos de coagulação e interações com o cateter (COWGILL \& LANGSTON, 1996).

De acordo com a literatura (FISCHER et al., 2004), a maioria dos animais requer cateter de $15 \mathrm{~cm}$ de comprimento, ou maior, para que seja alcançado adequado fluxo sanguíneo durante a HD. Para cães com mais de $10 \mathrm{~kg}$, cateteres de 11,5 French são usados freqüentemente nos acessos agudos. O uso de cateter de 8 French X 16” (16cm), como o deste estudo, permitiu adequado fluxo sanguíneo nos cães estudados, nos quais o peso corpóreo variou entre 6 e $20 \mathrm{~kg}$, de acordo com ELLIOT (2000), que indica cateteres de 8 French na obtenção de grandes volumes de sangue, para adequado fluxo sanguíneo durante a HD.

Os cateteres de poliuretano, usados no acesso vascular temporário, são pouco flexíveis e propícios a obstruções, danos físicos e dobraduras. Uma vez que a trombose do cateter impede o fluxo sanguíneo extracorporal, reduz a eficiência dialítica (ELLIOT, 2000) e predispõe a infecção (BESARAB \& RAJA, 2003), sua ocorrência implica a substituição do mesmo (ELLIOT, 2000). Porém, quando necessária, a substituição pode ser realizada com uso de guia metálico, sem necessidade de punção adicional da veia para sua implantação (COWGILL \& LANGSTON, 1996), resultados similares aos do atual estudo.

A troca do cateter de duplo lúmen através de guia metálico não aumentou a ocorrência de infecções nos animais estudados, de acordo com o descrito no homem por OLIVER et al. (2000). A inserção de um cateter com um fio-guia está associada a menor desconforto e taxa de complicações mecânicas significativamente menores do que aqueles percutaneamente inseridos em um novo local (O’GRADY et al., 2002). Este tipo de cateter deve ser utilizado exclusivamente para a HD, e só deve ser manuseado por equipe treinada, impedindo injeções inadvertidas de heparina ou contaminação bacteriana (ELLIOT, 2000). Observou-se, no presente estudo, que a execução de curativo e cuidado de higiene no local de inserção do cateter minimizou a ocorrência de infecções.

No homem, a incidência de bacteremia ou infecção no local de puntura do cateter é baixa até três semanas após a colocação do mesmo na veia jugular interna. Após este período, há aumento considerável nos riscos de infecção (OLIVER et al., 2000). Na atual pesquisa, apesar de não terem sido realizados testes de cultura bacteriana, não foram observados leucocitose ou sinais clínicos que indicassem a presença de infecção em nenhum dos cães durante todo o período de permanência do cateter, que chegou a até 30 dias nos cinco cães sobreviventes.

Infecções relacionadas ao acesso vascular são descritas como complicação comum em cães (ELLIOT, 2000; FISCHER et al., 2004). Entretanto, dados clínicos apresentam baixa especificidade (febre) e 
sensibilidade (descarga purulenta pelo sítio de inserção) na detecção de infecções. Até 55\% dos cateteres de hemodiálise estão colonizados sem associação com manifestações clínicas (O’GRADY et al., 2002), o que pode explicar a ausência de infecções relacionadas ao acesso vascular no presente estudo, uma vez que foram considerados apenas os dados clínicos. Também os cateteres de poliuretano, como os usados neste estudo, estão associados com menor ocorrência de complicações infecciosas que cateteres feitos de polivinil ou polietileno (O’GRADY et al., 2002).

Apesar de os cães deste estudo apresentarem trombocitopenia e aumento nos valores do TCA, não foram observados sangramentos no ponto de inserção do cateter. Também animais portadores de IRA apresentam uma maior tendência a sangramentos. Sendo assim, o acesso vascular deve ser realizado da maneira menos invasiva possível (COWGILL, 1995). A técnica de implantação percutânea utilizada neste estudo (MENESES et al., 2002) evitou a realização da flebotomia, e não foi associada à ocorrência de hemorragias, mostrando-se satisfatória.

\section{CONCLUSÕES}

O acesso vascular temporário com cateter de duplo-lúmen não-tunelizado, inserido na veia jugular externa, é eficiente em promover fluxo sanguíneo adequado para HD e apresenta baixa incidência de complicações, podendo ser indicado como forma de acesso vascular para HD em cães com IRA.

\section{AGRADECIMENTOS}

À Unidade de Diálise da Unesp, Botucatu, pelo suporte financeiro desta pesquisa.

\section{COMISSÃO DE ÉTICA NA EXPERIMENTAÇÃO ANIMAL}

Os procedimentos com animais seguiram estritamente as normas do COBEA (Comitê Brasileiro de Experimentação Animal, lei n. 6.638 de 8 de maio de 1979) e foram aprovados pela Comissão de Ética na Experimentação Animal da Unesp, Botucatu (processo 160/2000, de 27 de novembro de 2000).

\section{REFERÊNCIAS}

AGRAHARKAR, M. et. al. Pan hemispheric infarction: a complication of cuffed catheter. Southern Medical Journal, Birmingham, v.96, n.2, p.194-197, 2003.

BESARAB, A.; RAJA, R.M. Acesso vascular para hemodiálise. In: DAUGIRDAS, J.T.; ING, T.S. Manual de diálise. 3.ed. Rio de Janeiro: Medsi, 2003. Cap.4, p.68-102.
BLAKE, P.G. et. al. The use of dual lumen jugular venous catheter as definitive long term access for haemodialysis. International Journal of Artificial Organs, Milano, v.12, n.1, p.26-31, 1990.

BONNET, J.M. et al. Adaptation d'une technique d'hémodialyse à l'espèce canine. Recueil de Médecine Vétérinaire, Paris, v.170, n.12, p.797-809, 1994.

BRANT, J.R.A.C. Estudo comparativo entre a heparina sódica e a heparina de baixo peso molecular em cães submetidos a hemodiálise. 2002. 85f. Dissertação (Mestrado em Clínica Veterinária) - Curso de Pós-graduação da Faculdade de Medicina Veterinária e Zootecnia, Universidade Estadual Paulista Júlio de Mesquita Filho, Unesp, Botucatu.

COWGILL, L.D. Application of peritoneal dialysis and hemodialysis in the management of renal failure. In: OSBORNE, C.A.; FINCO, D.R. Canine and feline nephrology and urology. Baltimore: Williams \& Wilkins, 1995. Cap.31, p.573596.

COWGILL, L.D.; LANGSTON, C.E. Role of hemodialysis in the management of dogs and cats with renal failure. Veterinary Clinics of North America: Small Animal Practice, Philadelphia, v.26, n.6, p.1347-1378, 1996.

COWGILL, L.D.; ELLIOT, D.A. Insuficiência renal aguda. In: ETTINGER, S.J.; FELDMAN, E.C. Tratado de medicina interna veterinária. 5.ed. Rio de Janeiro: Guanabara Koogan, 2004. Cap.168, p.1701-1721.

DANTAS, A.F. et. al. I ntoxicação experimental por gentamicina em cães. Ciência Rural, Santa Maria, v.27, n.3, p.451-456, 1997.

DICE, K.M. et al. Tratado de anatomia veterinária. Rio de Janeiro: Guanabara Koogan, 1990. 567p.

ELLIOT, D.A. Hemodialysis. Clinical Techniques in Small Animal Practice, Orlando, v.15, n.3, p.136-148, 2000.

FISCHER, J.R. et al. Veterinary hemodialysis: advances in management and technology. Veterinary Clinics of North America: Small Animal Practice, Philadelphia, v.34, n.4, p.935-967, 2004.

IKEDA, S.; CANZIANI, M.E.F. Acesso vascular para hemodiálise. In: AJZEN, H.; SCHOR, N. Guias de medicina ambulatorial e hospitalar. São Paulo: Manole, 2002. p.231240 .

JAIN, N.C. Essentials of veterinary hematology. Philadelphia: Lea \& Febiger, 1993 . 417p.

LANGSTON, C. et. al. Applications and outcome of hemodialysis in cats: a review of 29 cases. Journal of Veterinary Internal Medicine, Philadelphia v.11, n.6, p.348355, 1997.

MELCHERT, A. Tratamento hemodialítico da insuficiência renal aguda por gentamicina em cães. 2005. 65f. Tese (Doutorado em Fisiopatologia em Clínica Médica) - Curso de Pós-graduação em Medicina, Universidade Estadual Paulista Júlio de Mesquita Filho, Unesp, Botucatu. 
MENESES, A.M.C. et al. Acesso vascular para hemodiálise em cães utilizando cateter de duplo lúmen. In: CONGRESSO BRASILEIRO DE CLÍNICOS VETERINÁRIOS DE PEQUENOS ANIMAIS, 23., 2002, Brasília, DF. Anais... Brasília: Anclivepa- DF, 2002. CD-ROM. 1 CD-ROM para uso em PC.

MORRISON, D.F. Multivariate statistical methods. São Paulo: Mc Graw-Hill, 1990. 450p.

O'GRADY, N.P. et al. Guidelines for the prevention of intravascular catheter-related infections. Morbidity and Mortality Weekly Report, Atlanta, v.51, n.10, p.1-29, 2002.

OLIVER MJ. et al. Risk of bacteremia from temporary hemodialysis catheters by site of insertion and duration of use: a prospective study. Kidney International, New York, v.58, n.6, p.2543-2545, 2000.
TESIO, F. et. al. Double catheterization of the internal jugular vein for hemodiálisis: indications, techniques, and clinical results. Artificial Organs, Cambridge, v.18, n.4, p.301-304, 1994.

VEADO, J.C.C. et al. Hemodiálise na medicina veterinária. Cadernos Técnicos de Veterinária e Zootecnia, Belo Horizonte, v.32, p.98-103, 2000.

WEIJMER, M.C. et al. Compared to tunnelled cuffed haemodialysis catheters, temporary untunnelled catheters are associated with more complications already within 2 weeks of use. Nephrology Dialysis Transplantation, Oxford, v.19, n.3, p.670-677, 2004.

WOJCIESZEK, E. et al. The complications of the use of hemodialysis catheter in central vein of the upper part of body - own experience. Wiadomósci Lekarskie, Katowice, n.34, p.197, 2002. 